\title{
Human Identification using Histogram of Oriented Gradients (HOG) and Non-Maximum Suppression (NMS) for ATM Video Surveillance
}

\author{
Sanjeevkumar Angadi ${ }^{1}$, Suvarna Nandyal ${ }^{2}$ \\ ${ }^{1}$ Assistant Professor, Department of Computer Science and Engineering, \\ MAEER's MIT College of Railway Engineering and Research, Barshi, India \\ ${ }^{2}$ Professor, Poojya Doddappa Appa College of Engineering, Kalaburagi, India \\ Correspondence should be addressed to Sanjeevkumar Angadi; angadi.sanjeevkumar@gmail.com
}

Copyright (C) 2021 Made Sanjeevkumar Angadi et al. This is an open access article distributed under the Creative Commons Attribution License, which permits unrestricted use, distribution, and reproduction in any medium, provided the original work is properly cited.

\begin{abstract}
Today video surveillance is the scorching topic in the research field of Computer Vision. Around 400 million surveillance cameras are used in various sectors which merely act as blind and record videos for postincident analysis. With the increase in crimes around the world, video surveillance plays a key role in identifying anomaly activities in a day to day application. One such application is a robbery in Automated Teller Machine (ATM). Identifying and tracking unlawful human activities is a challenging task in the ATM video surveillance system. Thus, an effective Human detection method using computer vision and image processing is proposed to create phenomenal results. The proposed approach, incorporate the most acclaimed Histogram of Oriented Gradient (HOG)Support Vector Machine (SVM) with a combination of Adaptive Background Generation Model and NonMaximum Suppression (NMS) algorithm to detect human with appropriate results in a video sequence. The experimentation result of the proposed method is applied on ATM Video Surveillance, real-time dataset. The performance analysis is evaluated by considering the average value of metrics such as the number of an input frames, ground truth elements and frames with human identified which acquired $97 \%$ of accuracy.
\end{abstract}

KEYWORDS- Human Detection, Background Subtraction, Support Vector Machine (SVM), NonMaximum Suppression (NMS), Histogram of Oriented Gradients (HOG), Video Surveillance

\section{INTRODUCTION}

Today's most significant research area in the field of the computer community is Computer Vision. To solve challenging tasks of the real-world in the domain of computer vision, science and technology play a vital role in extracting and processing the information. As a scientific discipline, the information extracted from images which are concerned with computer vision has the theories of artificial systems. Image data which are extracted can have many forms, such as, multiple views of cameras, video sequences and medical scanner with multi-dimensional data. Today, Image Processing and Computer Vision techniques are widely used in industries for automation, biological science for developing bioinformatics, video surveillance for analysis of real-time data, medical science and many more. Recent development disciplines of computer science are Image processing, Pattern Recognition, Artificial Intelligence and Neural Networks have promised the technological support required to tackle the various issues in computer vision. In recent years, the research on video surveillance systems is being witnessed with Closed Circuit Television (CCTV) cameras installed in almost every security-critical location. The need for video analysis systems for ensuring safety and security is empowered and realized by smart video surveillance systems in place. The innovations in video processing with advances in video processors coupled with video analysis software algorithms have produced very efficient systems, able to find target video patterns like motion, dynamic gestures, etc. Various application such as anomalies detection, object detection and tracking, traffic monitoring, theft identification, postincident analysis etc. use video surveillance systems as a security concern [21]. One such application is ATM Surveillance. ATMs have reported worldwide with many Criminal activities, such as fraudulent cards, magnetic strips, robberies, abnormal behavior like fighting, snatching of money, etc., [17]. ATM crimes are reported not only in developing countries but in developed countries as well [1]. With the existence of today's video surveillance system, as reported in news, thieves are not afraid of these techniques, because these systems record the happenings and are used later for the forensic investigation of the crime scene and do not help much in the prevention of crimes. Despite being a potential research area a very few traces of critical reviews have been found for the available articles on ATM crime detection [4] [5] [9] [18] [20] using a surveillance camera. Therefore, an instant detection of criminal activities using surveillance cameras has become a demanding issue to ensure a secure ATM environment. To overcome these demanding issues, we have proposed an automation system in which the initial stage of automation is human identification. The proposed model is a novel method for human identification which involves different steps like Support Vector Machine (SVM) classifier, Histogram of 
Oriented Gradients (HOG), and Non-Maximum Suppression (NMS) algorithm for an ATM-based application.

\section{LITERATURE SURVEY}

The need to develop a novel system that can monitor video data and subsequently analyze it for identifying abnormalities that may compromise public safety has been experienced with the comparative analysis of most of the existing work is summarized to design a robust algorithm and to address the gaps in the literature.

Hae-Min Moon et.al [10] say that videos recorded through a surveillance system when exposed have an open issue with privacy protection. To overcome such privacy protection involved with humans, a human identification technique is necessary. The main aim of this proposed model is to identify humans based on clothing-color and height information required for a surveillance system based on a smartcard. By using the octree-based color quantization technique on clothing, a representative color is extracted, and based on geometrical information in an image, height is extracted. The proposed model completely depends on the smartcard reader for identification and works only for the indoor environment.

Win Kong et.al [20] state about new challenges that have opened up for researchers in the field of a video surveillance system for human identification. The author has developed a new method for human identification and tracking which is based on Hand Silhouette. The proposed model has developed a novel technique that is based on Euclidean distance profile calculation. The proposed algorithm is a simple one used in human shape representation. The challenges that can be considered for better performance is to create a skeleton with more key points to describe other human body parts.

Alok K. Singh Kushwaha et.al [3] says that the multiple object/human detection and tracking is an open challenge for video surveillance researcher. The problem has arisen due to variation in the poses of a human object, poor lighting conditions, size, clothing shape, etc. The proposed model has developed a novel technique for multiple human detection and tracking in a video. Initially, a trained classifier is used for object detection which is based on Haar-like features that are considered from training image sets. A trained detector helps in the detection of Human objects and a practical filter is used for tracking. The proposed model works only with an outdoor environment with few conditions like poor lighting and human poses in consideration.

Swapnil V. Tathe et.al [14] speak about a challenge for human detection and tracking in real-time applications. Previously, research for authentication of a person using biometric has reached far, but the tracking of humans has not gained much importance in real-time. The proposed model has two parts, one is human detection and another is tracking. Face detection and Eye detection method are used for Human detection in this proposed model. As a part of face detection, the face of a human being represents the most important information about the individual. For person identification, biometric features of eyes can be used. In the proposed model, as eye detection methods restrict to the face region, there is always a performance dependency between eye detection methods and face detection methods.

Cancela B et.al [6] have given an approach for Human detection and tracking can be accomplished by different algorithms as well as specially designed architectures exploiting silhouettes of the human body. The occlusions in the frames of video pose difficulty in detection and tracking humans. During occlusion, a part of the target object is hidden by the occluding object for a time either totally or partially. The features of the target object thus cannot be extracted in that case continuously. Re-identification of the target in multiple cameras, or during total or partial occlusion is to be ensured. The structure and shape of the target persons cannot be recognized for that duration.

Sriram K.V et. al [13] proposed a model for human detection and tracking in a video surveillance system. The proposed approach has two steps; one to detect the person using Gaussian Mixture Model and another is used to track the detected person using the Kalman filter. By performing the detection operation on down-sampled video, the processing time is reduced to detect the person. PapoulisGerchberg method is used to reconstruct the original size of the video after the person is detected.

H.-C. Lu et.al [11] has proposed a model for real-time facial expression recognition. The proposed method aims to recognize different facial expressions based on pixelpattern-based texture features [PPBTF]. For characterizing facial texture, an image of a gray-scale is transformed into pattern maps where edges and lines are used. The experiment results of the proposed work are quite effective, speedy, and robust to illumination.

Ehab Salahat et.al [8] described the modern advances in feature detection and description algorithms developed for video surveillance purposes. The fundamental concepts have been discussed initially. A comparison of these algorithms for their functional abilities, performance, advantages, and disadvantages has been taken up. The Maximally Stable External Regions algorithm and the Scale Invariant Feature Transform algorithms are used for reporting the algorithmic complexity and derivatives. From the literature survey, it is found that most of the work is carried out on human detection on simple applications in video surveillance, facial expression identification for different features considering the motion, movements of humans in the crowded area for tracking of behavior. Hence, to develop an Automatic video analysis technology a smart surveillance system is proposed which can aid the human operator in real-time threat detection tasks and also in multiscale tracking systems. 


\section{PROPOSED SYSTEM}

The current surveillance system, consumes a lot of resources such as memory, due to nonstop recording. They are efficient but tracking the events when required is a timeconsuming task. Due to the negligence of humans, there may be a miss classification of events. To avoid all these challenges, we have proposed an automation system for human identification as an initial stage of research. The proposed approach involves various steps such as background subtraction, morphological operation, foreground image, and people detection. Figure 1 shows the proposed block diagram for Human Identification based on ATM Application.

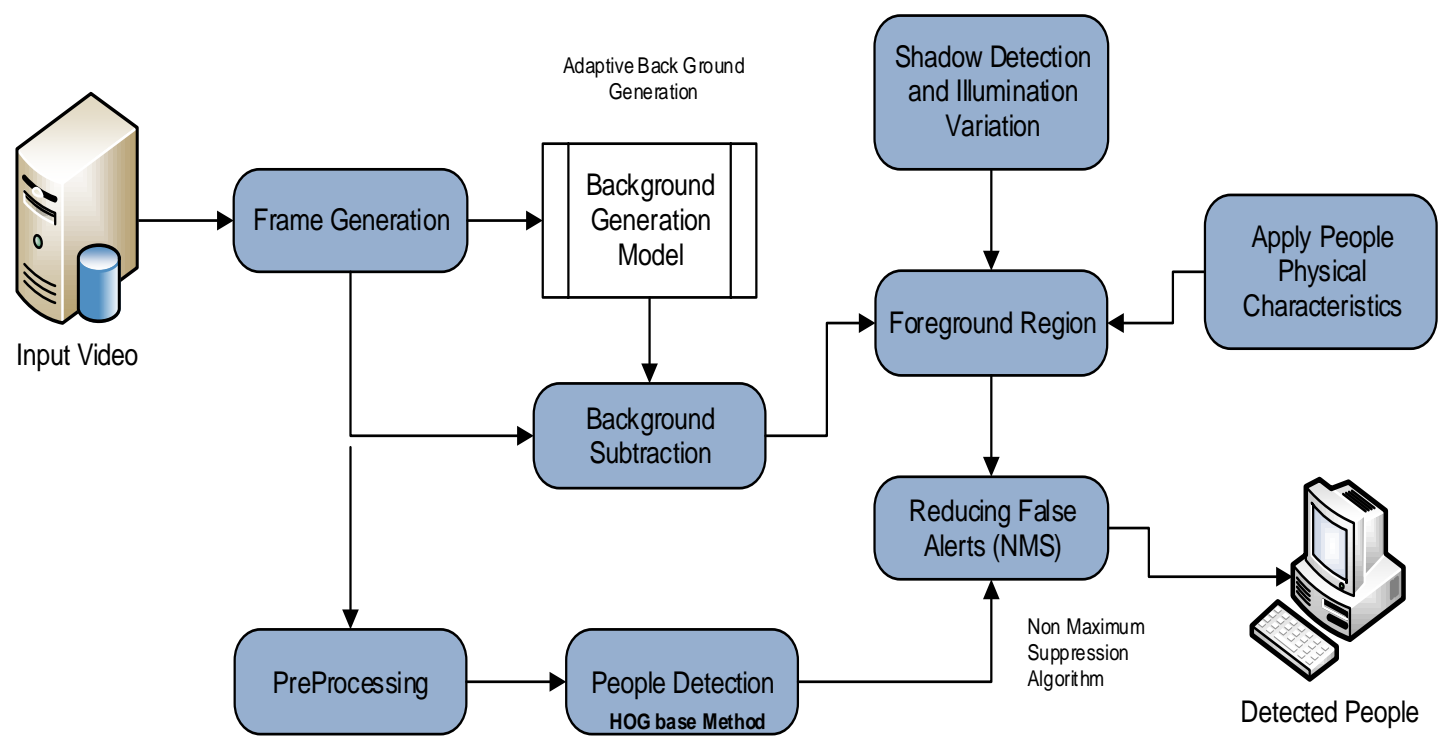

Fig. 1: Proposed System for Human Identification

The following steps are the procedure for human identification under video surveillance:

Step 1: The input video is converted to frames and the frames are in RGB color space.

Step 2: Convert each RGB image into its grayscale form and apply preprocessing.

Step 3: Background subtraction is carried out using the adaptive background generation method, to achieve the static background.

Step 4: Foreground mask and Foreground image are computed using Morphological operation.

Step 5: Histogram of Oriented Gradient (HOG) based method is used for People Detection. Bounding box and Validation Scores are computed.

Step 6: A false result is suppressed using the Non Maximum Suppression (NMS) algorithm to acquire the accurate result of Human Identification.

\section{A. Pre-Processing Of Input Frames}

The Pre-processing step is an important process in image processing used to remove all the external noise in the given input frames. The process used here is to convert an RGB image into grayscale and Resizing of an image is considered, based on the input frames.

\section{B. Background Generation}

For detecting foreground objects as well as to model the background, the background subtraction method plays a crucial role in any human detection phase. Initially, all the input frames are averaged to get the trajectory learning of background from the moving objects that are considered in the input frames. Based on pixel density and threshold value, an adaptive based background generation algorithm is used to determine whether the current frame consists of any moving objects or not. During this process, the background input frame will be initialized as $\mathrm{P}[\mathrm{D}]$. The input frames are measured in several ways to get the proper background image. For example, consider the primary input frame and initialize the primary frame into the subtraction of the consecutive input frame and organize the frames according to the moving object. The subtraction method then converts each incoming input image into a binary image and masking is done image by image for each frame. The background segmentation is to involuntarily produce a binary mask that splits the cluster of pixels into 2 sets, foreground, and background pixels [16].

The subtraction of the input frame is done as follows:

$\mathrm{P}[\mathrm{F}(\mathrm{g})]=\mathrm{P}[\mathrm{I}(\mathrm{g})]-\mathrm{P}[\mathrm{D}]$

$|\mathrm{P}[\mathrm{B}(\mathrm{g})]|-|\mathrm{P}[\mathrm{B}(\mathrm{g}+1)]|>$ Value of Threshold

$\mathrm{P}[\mathrm{B}(\mathrm{g})]$ background input frame, $\mathrm{P}[\mathrm{I}(\mathrm{g})]$ iteration of each input frame and $\mathrm{P}[\mathrm{D}]$ is background input frame of fixed reference. $\mathrm{P}[\mathrm{B}(\mathrm{g}+1)]$ is video frame of next input.

\section{Foreground Image}

The foreground mask and foreground image are generated after the step of background modeling. It will classify the necessary background and foreground object by identifying its pixel value from the input image. A simple foreground image is acquired using absolute differences between two incoming images. A converted binary image is obtained from the foreground image. The input image with 
luminance greater than level with the value 1 and value 0 , replaces all the pixels from the output binary image. To this output binary image, an inbuilt Morphological Operation has been used to remove the small parts of connected components to achieve the foreground mask.

\section{Histogram of Oriented Gradients (HOG)}

The Histogram of Oriented Gradient (HOG) is a method for object recognition used in image processing and computer vision based on the properties of feature descriptors. The image local area has the distribution of gradient magnitude and gradient orientation dependent on the feature of HOG. For human/object detection, first, it extracts the features from a local image, through a training sample, and then classifies the object by Support Vector Machine (SVM) classifier. The good characterization of appearance and shape are described based upon HOG features [7]. In the research field of computer vision, it is one of the proven methods which are used for human identification/detection and has become a conventional method. A small connected region in an image is divided into cells. A histogram of the gradient is drawn from an image with a small cell that holds several pixels and for each of the pixels. For enhanced exactitude the HOG is contrast-normalized and it is achieved by calculating intensity over a larger area cell known as a block, then this value is used to normalize all cells within that block. Based upon variation in illumination and intensity, better performance is attained for a normalized result. Parameters such as photometric transformations except for object orientation and invariant to geometric are some of the advantages of HOG descriptors over other descriptors. In this proposed system, an inbuilt function vision.peopledetector has been used to detect upright people using HOG features. In an input image, people are identified based on features and a trained Support Vector Machine (SVM) classifier [2]. The method returns the M-by-4 matrix defining $M$ bounding boxes, where $\mathrm{M}$ represents the number of detected people. Each row of the output matrix contains a four-element vector, [x, $y$, width, height]. This vector specifies, in pixels, the upperleft corner and size, of a bounding box.

The computational approach of HOG as follows:

The image $\mathrm{I}(\mathrm{x}, \mathrm{y})$ is the gray value of $\mathrm{I}$ in $\operatorname{pixel}(\mathrm{x}, \mathrm{y})$.

i) By performing gray-scale normalization, different images with illumination variance are reduced, so that the intensity range for all the images is the same.

ii) For masking to be performed, horizontal gradient $\mathrm{mx}(\mathrm{x}$, y) and vertical gradient $\mathrm{ny}(\mathrm{x}, \mathrm{y})$ of every pixel are computed.

$\operatorname{mx}(\mathrm{x}, \mathrm{y})=\mathrm{I}(\mathrm{x}+1, \mathrm{y})-\mathrm{I}(\mathrm{x}-1, \mathrm{y})$

$\mathrm{ny}(\mathrm{x}, \mathrm{y})=\mathrm{I}(\mathrm{x}, \mathrm{y}+1)-\mathrm{I}(\mathrm{x}, \mathrm{y}-1)$

and then each pixel is computed for magnitude and

orientation of the gradient.

$$
\mathrm{G}(\mathrm{x}, \mathrm{y})=\sqrt{p x^{2}(x, y)+p y^{2}(x, y)}
$$

$\operatorname{Orient}(\mathrm{x}, \mathrm{y})=\arctan (\operatorname{mx}(\mathrm{x}, \mathrm{y}) / \mathrm{nx}(\mathrm{x}, \mathrm{y}))$

The $\left[-\frac{\pi}{2}, \frac{\pi}{2}\right]$ direction of the gradient evenly divides into nine bins. The magnitude of each pixel in the bin $\mathrm{Vx}(\mathrm{x}, \mathrm{y})$ given by:

$$
\mathrm{V}_{\mathrm{k}}(\mathrm{x}, \mathrm{y})=\left\{\begin{array}{c}
G(x, y), \alpha(x, y) \in \operatorname{bin}_{k} \quad 1 \leq \mathrm{k} \leq 9 \\
0, \alpha(x, y) \notin \operatorname{bin}_{k}
\end{array}\right.
$$

The final step is to collect the HOG descriptors covering the detection window from all blocks of a dense overlapping grid of blocks into a combined feature vector for use in the window classifier.

\section{E. Non-Maximum Suppression Algorithm}

Non-maximum suppression (NMS) is one of the postprocessing steps in computer vision applications [19]. Initially, in a sequence of image to-be-detected, a multiple quasi-targets are possible, but due to the impact of scale and traversal over a pixel, there can be a multiple Bounding Boxes (BB) in the same target, so it is necessary to suppress the false bounding boxes and have the accurate detection rate. Therefore, a non-maximum suppression algorithm is used always to find the exact identification in a given input image. To transform a smooth response map that triggers many imprecise object window hypotheses in the context of object detection, the ideal consideration is a single bounding-box for each detected object [12]

Computational is as follows:

$S_{i}=\left\{\begin{array}{c}S_{i}, \operatorname{iou}\left(M, b_{i}<N_{t}\right. \\ 0, \text { iou }\left(M, b_{i} \geq N_{t}\right.\end{array}\right.$

When Intersection over Union (IoU) is less than the threshold $\mathrm{Nt}$, the detection score is $\mathrm{Si}$; when IoU is greater than the threshold $\mathrm{Nt}$, the score is 0 . This process is applied recursively to the remaining bounding boxes.

\section{RESULTS AND DISCUSSION}

\section{A. Experimental Setup}

Performance analysis of the proposed work is carried out using a real-time ATM dataset [15]. The database consists of around 40 videos with normal and anomalies activities that take place in ATM surveillance. The dataset is constructed by creating a dummy ATM structure in an indoor environment by considering all the lighting conditions. For recording these videos we have used Nikon DSLR 3300 along with a set of tripods and the MATLAB tool is used for implementation.

\section{B. Performance Metrics}

The performance metrics used to evaluate the methods are based on the analysis of Number of Input Frames, Ground Truth Element, and Frames with Identified humans.

- The number of Input Frames: Input frames are extracted from given input videos by considering various parameters namely, bits per pixel, frame rate, and video format.

\section{Experimental Results}

This section discusses the results of the proposed approach by considering three different input videos samples, varying of duration, position of human, respectively.

\section{- Experimental Results Video 1}

Initially, frame extraction takes place by considering a set of parameters such as video format, frame rate and duration of an input video. The video format used here is RGB24 
and the frame rate of $15.016 \mathrm{~s}$. The number of images generated is 939 input images.

Figure 2 shows the results of the proposed approach by considering the video sample. Figure 2 (a), Figure 2 (b), and Figure 2 (c) show the input image 1, input image 2, and input image 3 of video sample 1. The Foreground Mask and
Image for Input images 1, 2, 3 are shown in Figure 2 (d), Figure 2 (e), and Figure 2 (f). Human detection for video frame 1 is depicted in Figure $2(\mathrm{~g})$, Figure $2(\mathrm{~h})$, and Figure 2 (i). The output image 1 , output image 2 , and output image 3 using NMS are shown in Figure 2 (j), Figure $2(k)$, and Figure 2 (l).

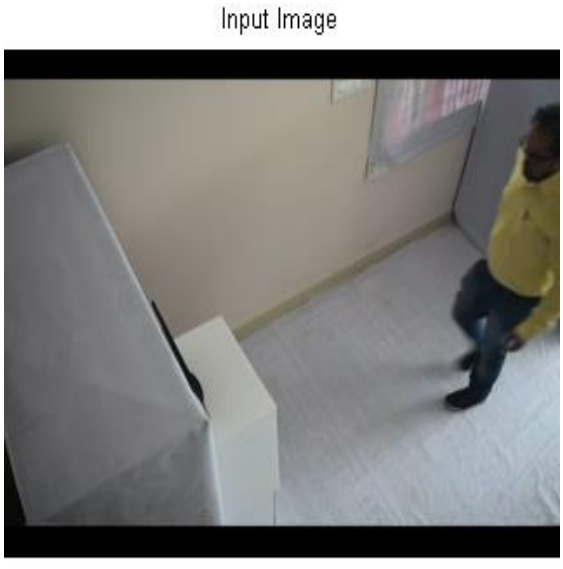

(a)

ForeGround Mask

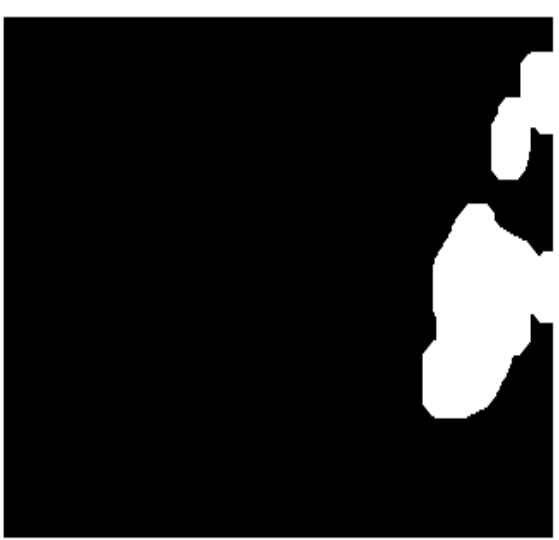

ForeGround Image

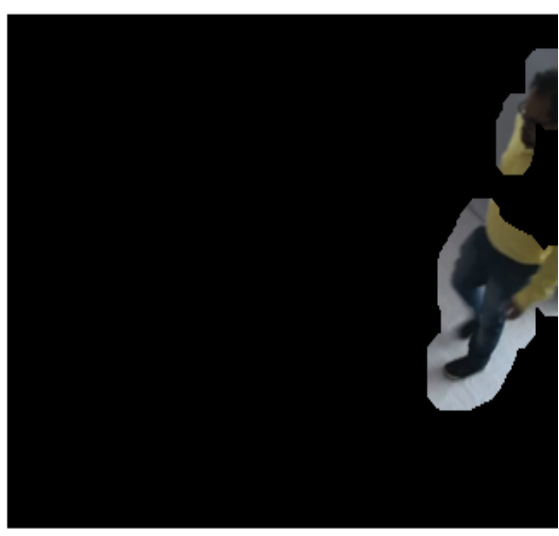

(d)

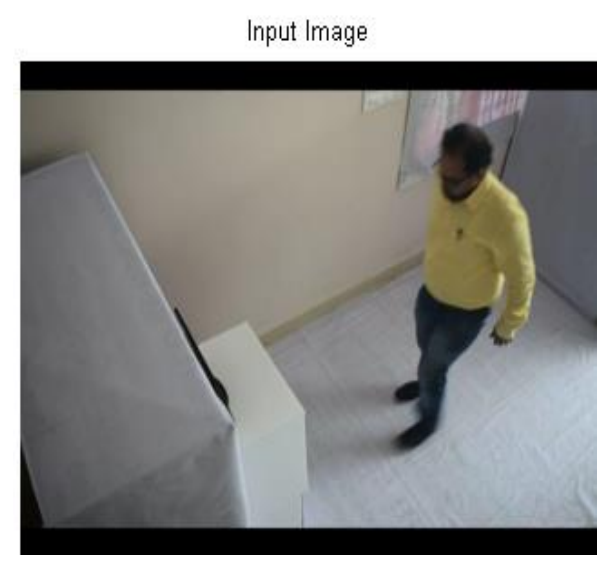

(b)

ForeGround Mask

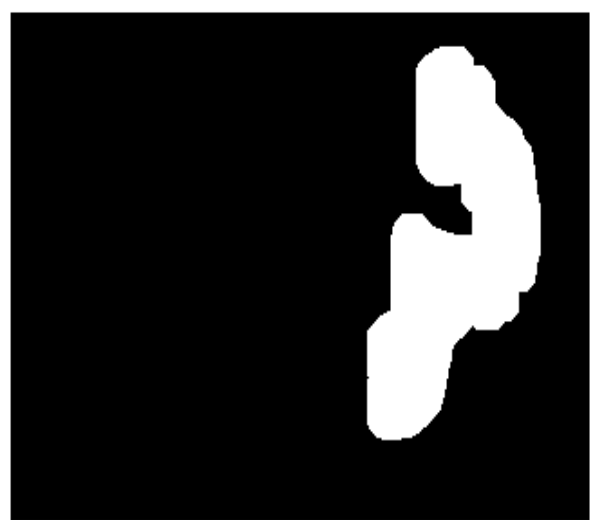

ForeGround Image

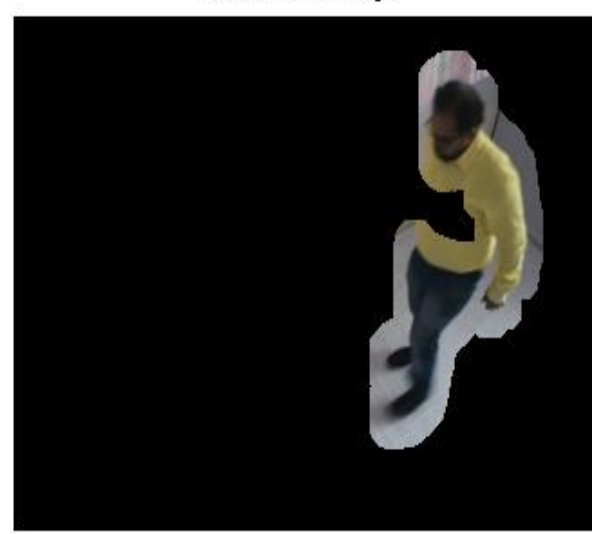

(e)

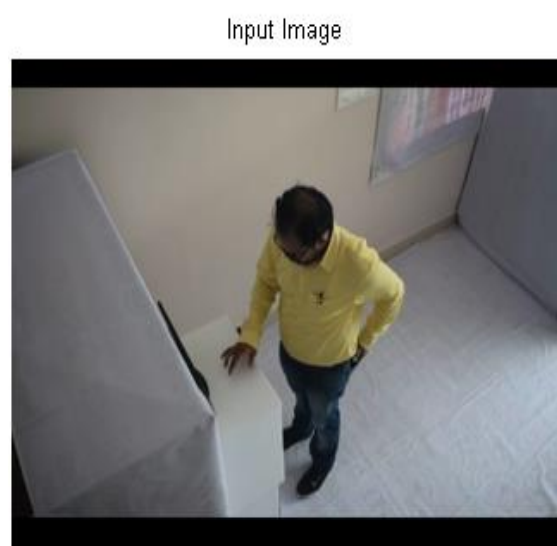

(c)

ForeGround Mask

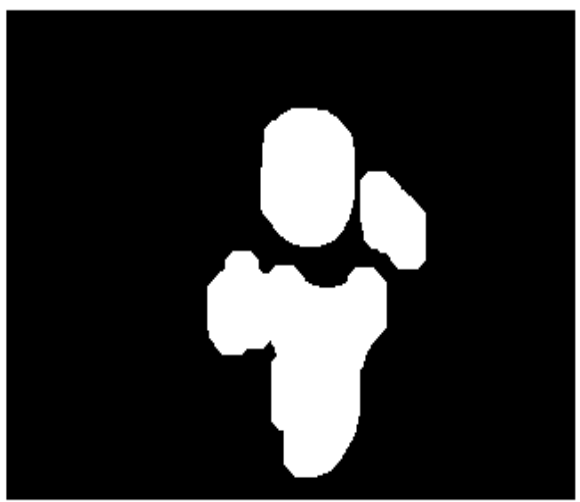

ForeGround Image

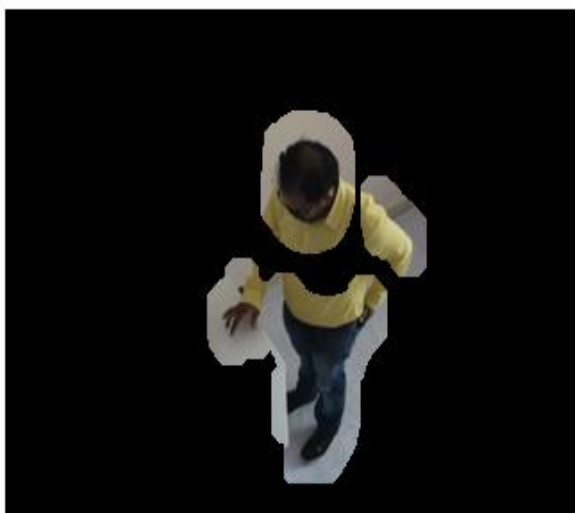

(f) 


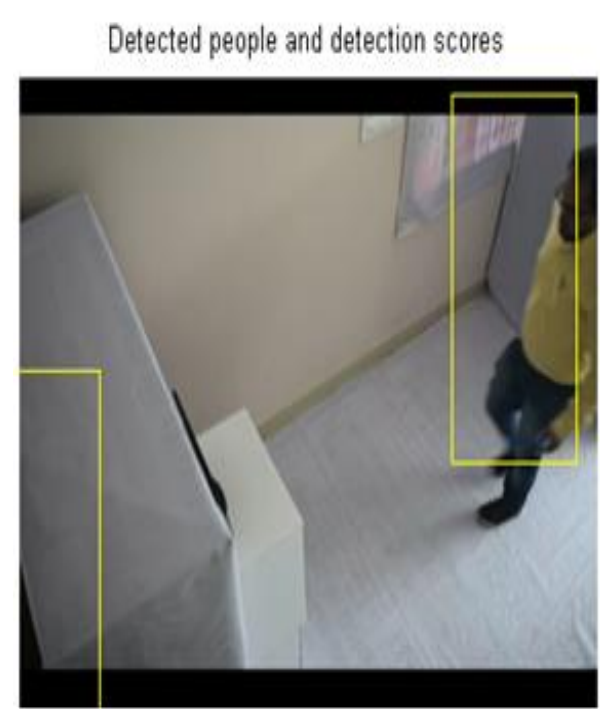

(g)

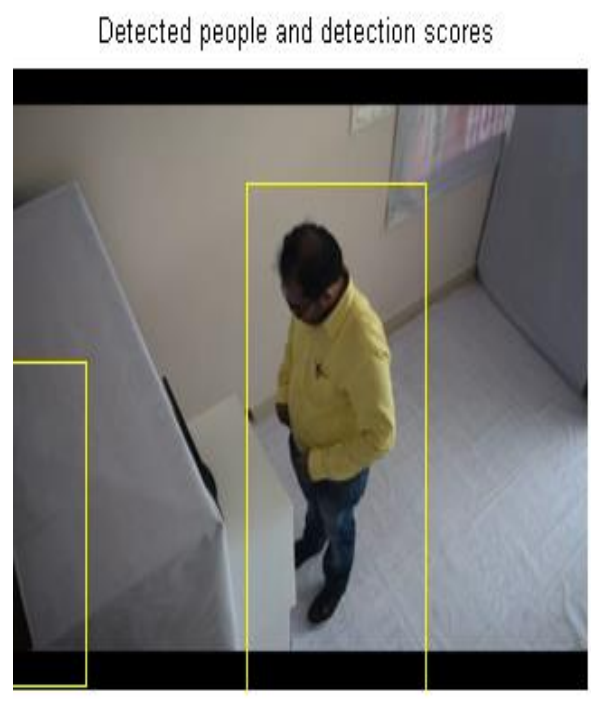

(h)

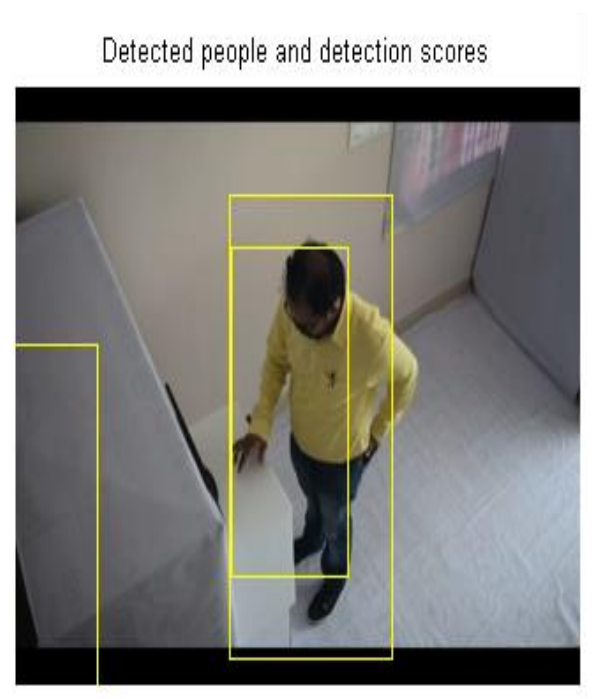

(i)

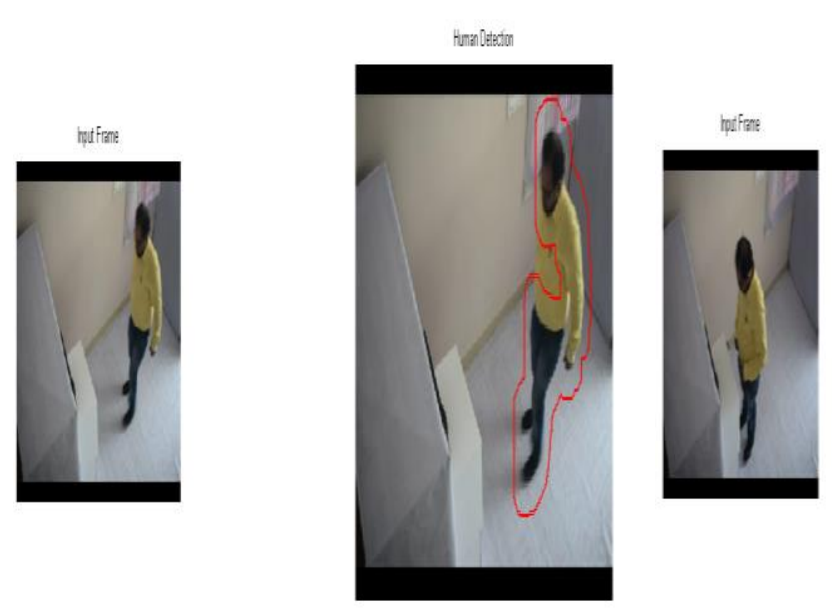

(j)

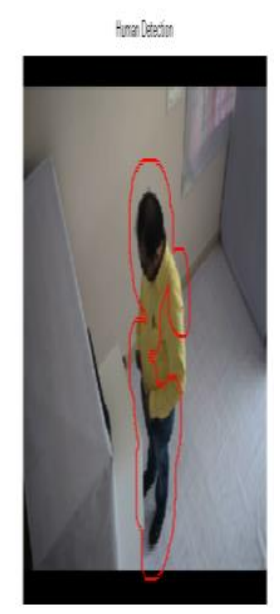

(k)

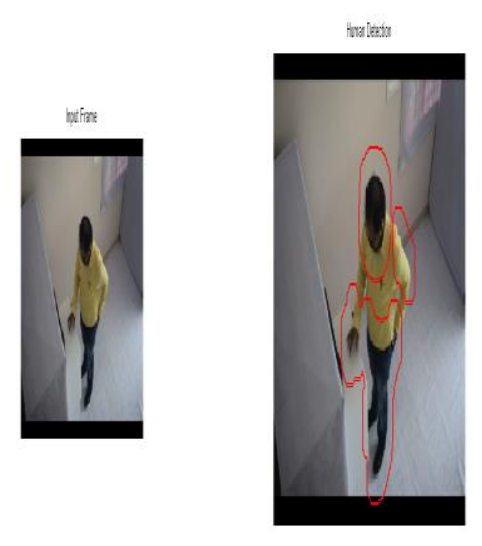

(l)

Fig. 2: Frames from sample video 1 (a) input image 1, (b) input image 2, (c) input image 3, (d) foreground mask and image of input image 1, (e) foreground mask and image of input image 2 (f) foreground mask and image of input image 3, (g) Detected People and Score of input image 1, (h) Detected People and Score of input image 2, (i) Detected People and Score of input image 3, (j) output image 1, (k) output image2, (l) output image 3.

\section{- Experimental Results of Sample Video 2}

Initially, input images are generated from the input video at a frame rate of $15.018 \mathrm{~s}$. The number of frames generated is 795 input frames.

Figure 3 shows the results of the proposed approach by considering the sample video 2. Figure 3 (a), Figure 3 (b), and Figure 3 (c) show the input image 1, input image 2, and input image 3 of video sample 2. The Foreground Mask and
Image for Input images 1, 2, 3 are shown in Figure 3 (d), Figure 3 (e), and Figure 3 (f). Human detection for video frame 2 is depicted in Figure 3 (g), Figure 3 (h), and Figure 3 (i). The output image 1, output image 2, and output image 3 using NMS are shown in Figure $3(\mathrm{j})$, Figure $3(\mathrm{k})$, and Figure 3 (l). 
International Journal of Innovative Research in Computer Science \& Technology (IJIRCST)

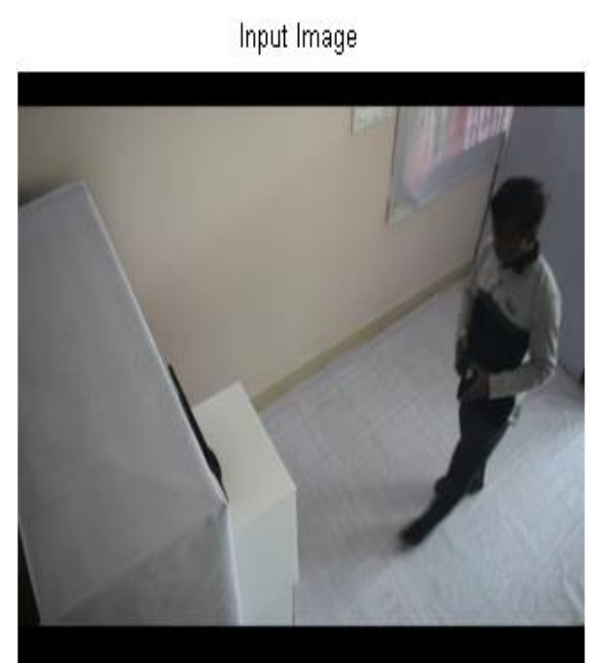

(a)

ForeGround Mask

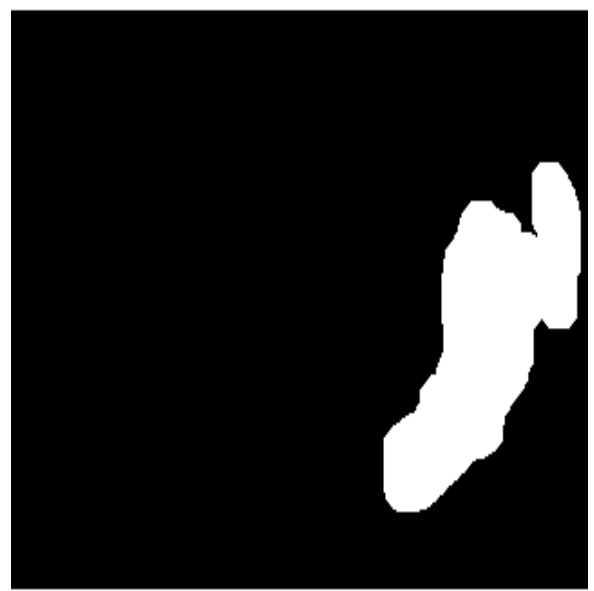

ForeGround Image

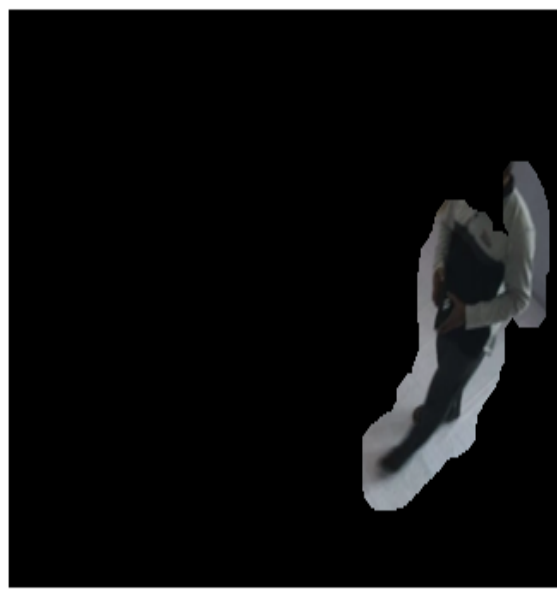

(d)

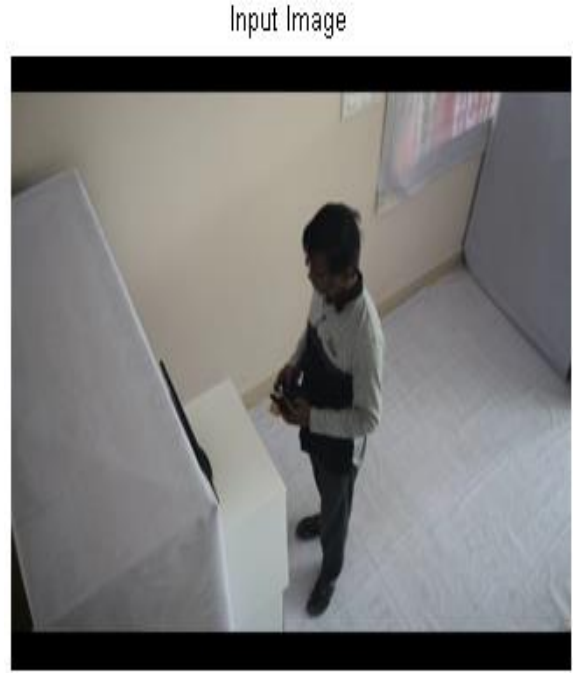

(b)

ForeGround Mask

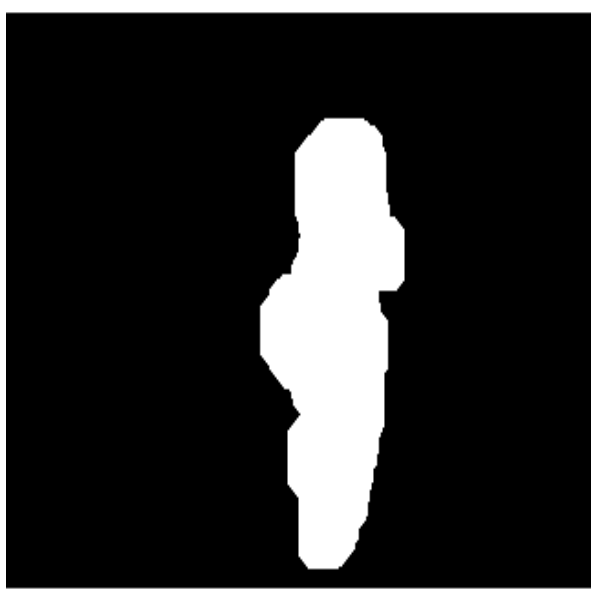

ForeGround Image

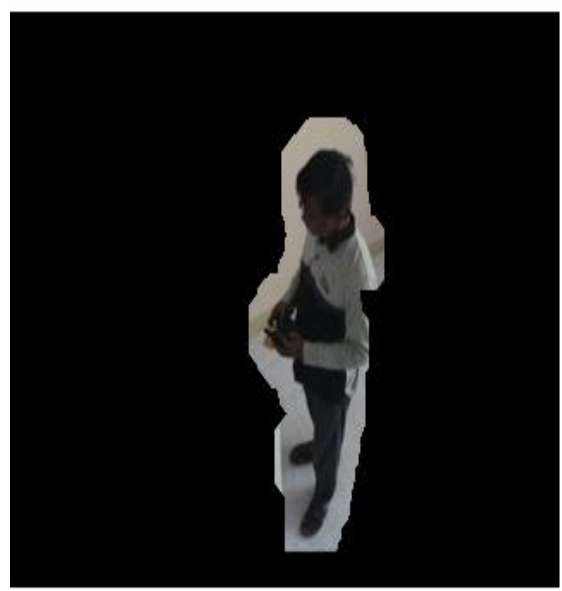

(e)

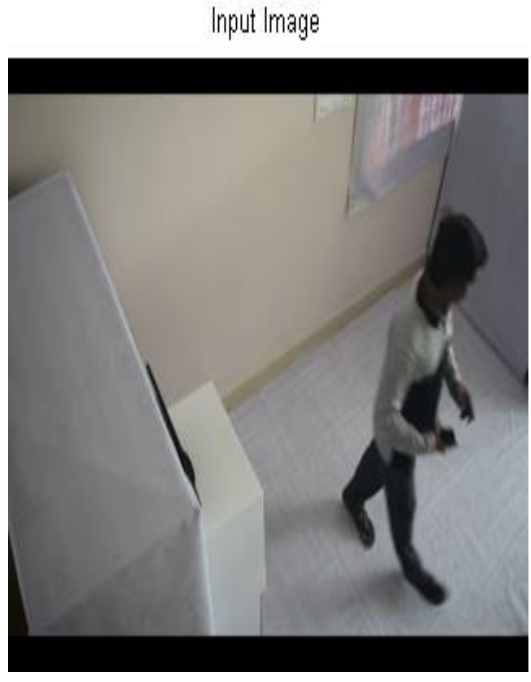

(c)

ForeGround Mask

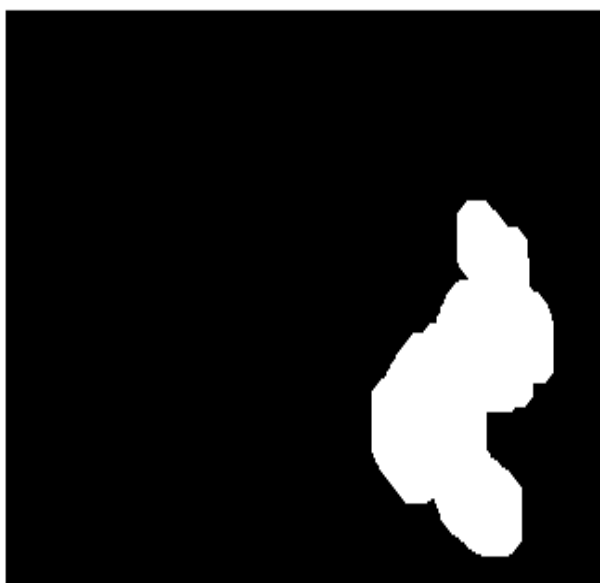

ForeGround Image

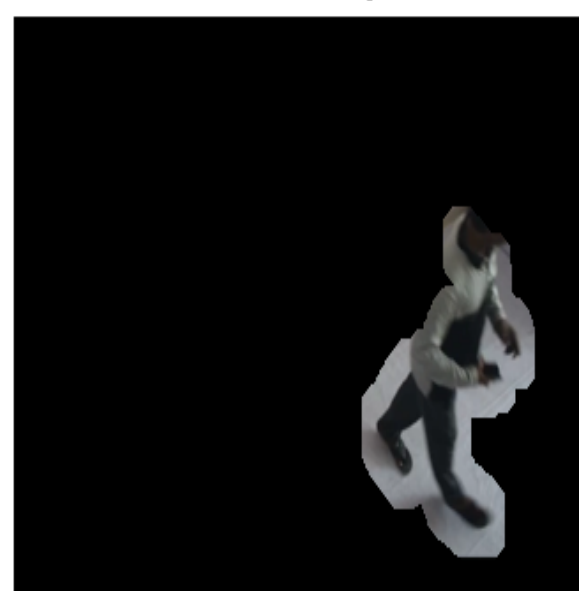

(f) 
Detected people and detection scores

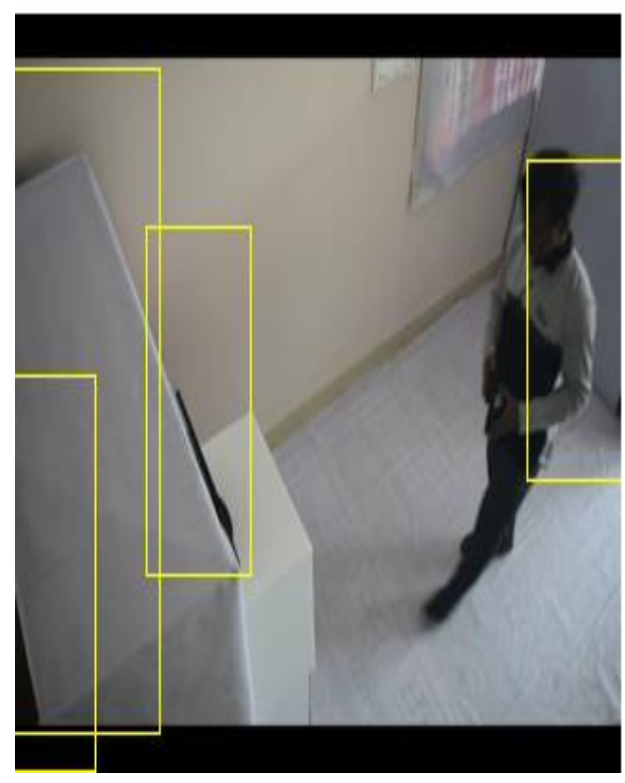

(g)

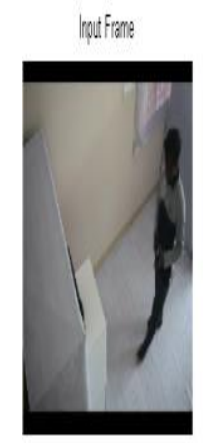

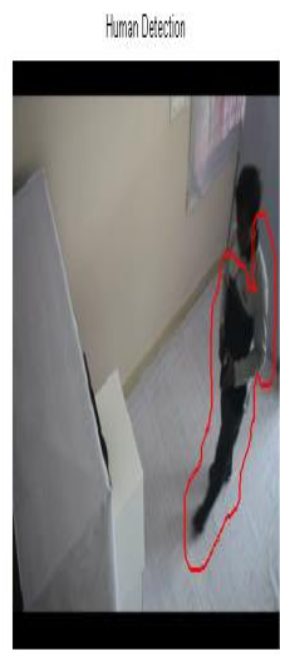

(j)
Detected people and detection scores

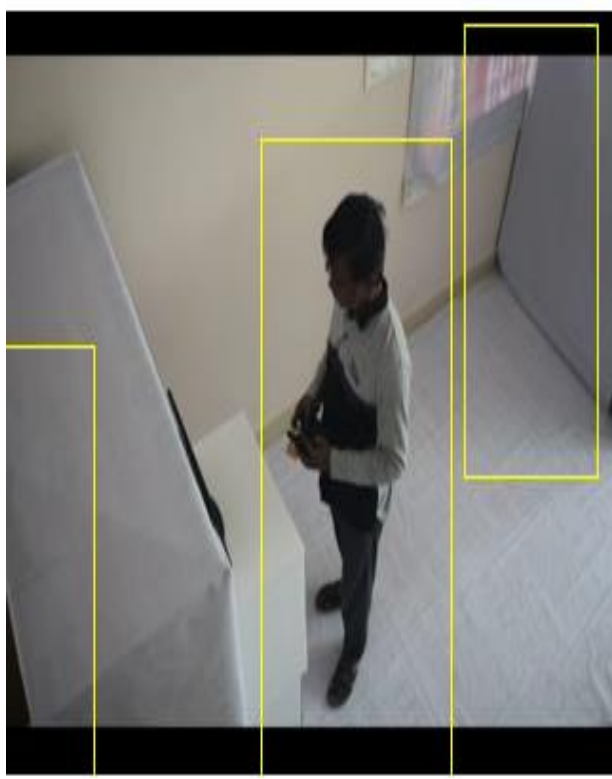

(h)

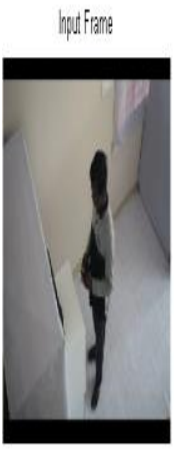

(k)
Detected people and detection scores

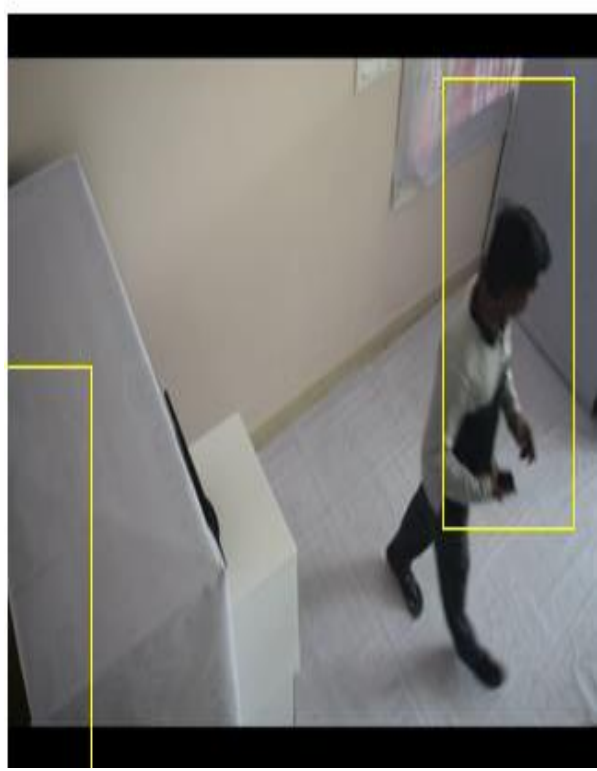

(i)
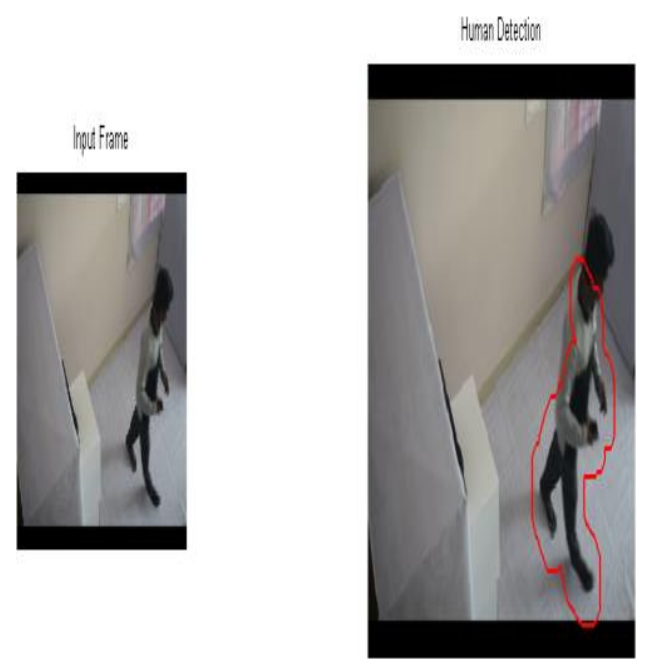

(l)

Figure 3: Frames from sample video 2, (a) input image 1, (b) input image 2, (c) input image 3, (d) foreground mask and image of input image 1, (e) foreground mask and image of input image 2 (f) foreground mask and image of input image 3, (g) Detected People and Score of input image 1, (h) Detected People and Score of input image 2, (i) Detected People and Score of input image 3, (j) output image 1, (k) output image2, (l) output image 3

\section{COMPARATIVE DISCUSSION}

This section describes the comparative analysis of the proposed approach by considering the average value of the metrics, as the number of input frames, ground truth element, and frames with identified humans using the test cases for video 1 , video 2 , video 3 , video 4 and video 5 . For video 1 , the input frames, ground truth element, and frames with an identified human for the proposed approach are given as, 939,880 , and 860 , respectively. For video 2 , the input frames, ground truth element, and frames with an identified human for the proposed approach are given as, 794, 737, and 722, respectively. For video 3, the input frames, ground truth element, and frames with an identified human for the proposed approach are given as, 836, 646, and 635 , respectively. For video 4 , the input frames, ground truth element, and frames with the identified human for the proposed approach is given as, 629, 518, and 505, respectively. For video 5, the input frames, ground truth element, and frames with an identified human for the proposed approach are given as, 952, 889, and 875, respectively. Based on the analysis for the above test case videos, the proposed approach identifies humans with an accuracy of $97 \%$ and is presented in the graph. Figure 4 shows the accuracy graph compared to the Ad boost algorithm. 
Table 1: Comparative analysis based on Ground Truth Element for Human Detection

\begin{tabular}{|c|c|c|c|c|c|c|}
\hline $\begin{array}{l}\text { Test } \\
\text { Case }\end{array}$ & $\begin{array}{l}\text { Number } \\
\text { of Frames } \\
\text { in Video }\end{array}$ & $\begin{array}{l}\text { Ground Truth } \\
\text { Element } \\
\text { (People Count) }\end{array}$ & $\begin{array}{l}\text { People } \\
\text { Detection } \\
\text { using } \\
\text { (Ad boost) }\end{array}$ & $\begin{array}{l}\text { People } \\
\text { Detection } \\
\text { using } \\
\text { (Proposed } \\
\text { Method) }\end{array}$ & $\begin{array}{l}\text { Accuracy } \\
\text { ( Ad boost) }\end{array}$ & $\begin{array}{l}\text { Accuracy } \\
\text { (Proposed) }\end{array}$ \\
\hline Video 1 & 939 & 880 & 2640 & 860 & $(2640 / 880) * 100=300 \%$ & $(860 / 880) * 100=97.72 \%$ \\
\hline Video 2 & 794 & 737 & 2211 & 722 & $(1474 / 737) * 100=200 \%$ & $(722 / 737) * 100=97.96 \%$ \\
\hline Video 3 & 836 & 646 & 1938 & 635 & $(1938 / 646) * 100=300 \%$ & $(635 / 646) * 100=98.29 \%$ \\
\hline Video 4 & 629 & 518 & 1554 & 505 & $(1554 / 518) * 100=300 \%$ & $(505 / 518) * 100=97.49 \%$ \\
\hline Video 5 & 952 & 889 & 2667 & 875 & $(2667 / 889) * 100=300 \%$ & $(875 / 889) * 100=98.42 \%$ \\
\hline
\end{tabular}

*The results of the Ad boost method are analyzed based on the bounding box drawn for people detection, which is an inbuilt function of Computer Vision Toolbox.

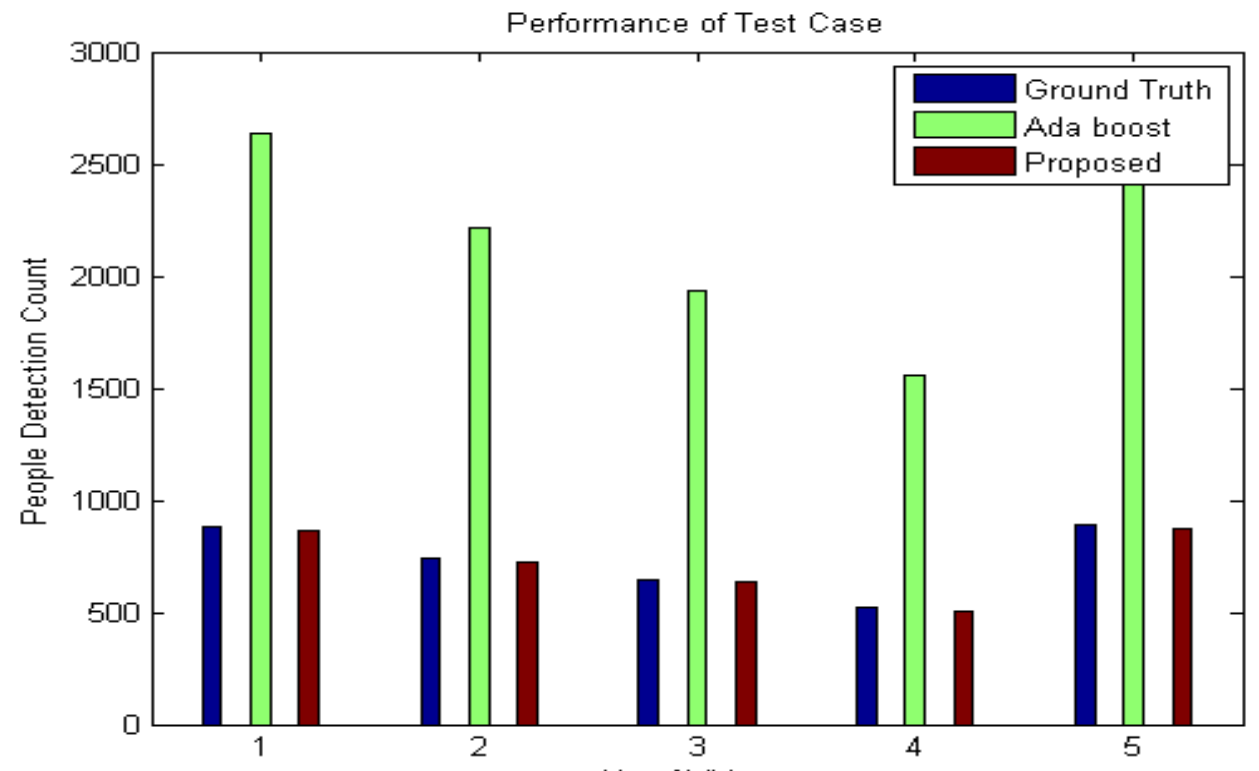

No of Videos

Figure 4: Comparative analysis based on Detection Frames

\section{CONCLUSION}

In this paper, a method is proposed to detect humans in a given video. This method consists of a Histogram of Oriented Gradients (HOG), Support Vector Machine (SVM) classifier, and Non- Maximum Suppression (NMS) algorithm for ATM Video Surveillance System. From the analysis, it can be seen that the proposed method has a good detection rate compared to the existing algorithms. The proposed approach achieves better performance for Human Detection with an average accuracy of $97 \%$.

\section{CONFLICTS OF INTEREST}

The authors declare that they have no conflicts of interest.

\section{REFERENCES}

[1] 100 thieves steal $\$ 13 \mathrm{~m}$ in three hours from cash machines across Japan. The Guardian, Japan. https://www.theguardian.com/world/2016/may/23/japan-cashmachine-100-thieves-steal-13m-dollars-three-hours accessed, 12 Jun 2016.

[2] Abdourahman Houssein Ahmed, Kidiyo Kpalma, Abdoulkader Osman Guedi, "Human Detection using HOGSVM, Mixture of Gaussian and Background Contours Subtraction", IEEE, International Conference on SignalImage Technology and Internet-Based Systems (SITIS), pp. 334-338, 2017.

[3] Alok K. Singh Kushwaha, Chandra Mani Sharma, "Automatic Multiple Human Detection and Tracking for Visual Surveillance System", IEEE/OSAIIAPR International Conference on Informatics, Electronics \& Vision, 2012.

[4] Another cash machine has blown up by robbers - this time Manchester Evening News, Barton Road, Stretford.https://www.manchestereveningnews.co.uk/news/gre 
ater-manchester-news/cashpoint-blown-up- stretford-atm 10537507, accessed, 2016.

[5] Bromwich, J.E.: A smash-and-grab heist in Pennsylvania: masked men steal an ATM. The New York Times, Pennsylvania.,https://www.nytimes.com/2016/12/01/us/asm ash-and-grab-heist-inpennsylvania masked-men-steal-anatm.html, accesses, 2016.

[6] Cancela, B., Ortega, M., Penedo, M.: "Multiple human tracking systems for unpredictable trajectories", Machine Vision and Applications, Vol.25, No.2, pp- 511-527, 2014.

[7] Chi-Chen Raxle Wang and Jenn-Jier James Lien, “AdaBoost Learning for Human Detection Based on Histograms of Oriented Gradients", Springer-Verlag Berlin Heidelberg, pp. 885-895, 2007.

[8] Ehab Salahat, Member, IEEE, and Murad Qasaimeh, Member, IEEE "Recent Advances in Features Extraction and Description Algorithms: A Comprehensive Survey", 2017.

[9] Graczyk, M.: Masked men steal lobby ATMs from 5Houston Marriott hotels. Daily Herald, Houston: http://www.dailyherald.com/article/20171214/news/3121498 23. accessed, Dec 2017.

[10] Hae-Min Moon, Sumg Bum Pan, "A New Human Identification Method for Intelligent Video Surveillance System", IEEE, 978-1-4244-7116-4/10/\$26.00 @2010.

[11] H.-C. Lu, Y.-J. Huang, Y.-W. Chen and D.-I. Yang, "Realtime facial expression recognition based on pixel-patternbased texture feature", Vol. 43 No. 17, 2007.

[12] Rasmus Rothe, Matthieu Guillaumin, and Luc Van Gool, "Non-maximum Suppression for Object Detection by Passing Messages Between Windows", Springer International Publishing Switzerland, pp. 290-306, 2015.

[13] Sriram K.V., R. H. Havaldar, "Human Detection and Tracking in Video Surveillance System", IEEE, 978-1-50900612-0/16/\$31.00 @2016.

[14] Swapnil V. Tathe and Sandipan P. Narote, "Real-Time Human Detection and Tracking", IEEE, 2013.

[15] Suvarna Nandyal, Sanjeevkumar Angadi, "Database Creation for Normal and Suspicious Behaviour Identification in ATM Video Surveillance", unpublished.

[16] Suvarna Nandyal, Sanjeevkumar Angadi, "Adaptive Background Generation Method for Automated Teller Machine (ATM) with an Integrated Video Monitoring System", unpublished.

[17] Tasriva Sikandar, Kamarul Hawari Ghazali, Mohammad Fazle Rabbi, "Multimedia Systems ATM crime detection using image processing integrated video surveillance: a systematic review" (c) Springer-Verlag, 2018.

[18] Thieves rip ATM out of a bank in St. Louis, Sask. CBC News, Saskatchewan, Saskatchewan, Canada.http://www.dailyherald.com/article/20171214/news/ 312149823.cbc.ca/news/canada/saskatchewan/atm-theftsask-1.3738849.2016.

[19] Tuan Q. Pham, "Non-maximum Suppression Using Fewer than Two Comparisons per Pixel", Springer-Verlag Berlin Heidelberg, pp. 438-451, 2010.

[20] Vanar, M.: Robbers blast ATM, escape with RM70,000. The Star Online, Kota Kinabalu., https://www.thestar.com.my/news/nation/2016/05/11/robber s- blast-atm-escape-with-rm70000/, accessed on, 2016.

[21] Venetianer, P. L., Deng, H. L., "Performance evaluation of an intelligent video surveillance system-A case study, Computer Vision", Image Understanding, vol. 114, no. 11, pp. 1292-1302, 2010

[22] Win Kong, Aini Hussain, Mohd. Hanif Md Saad \& Nooritawati Md. Tahir, "Hand Detection from Silhouette for Video Surveillance Application", IEEE 8th International Colloquium on Signal Processing and its Applications, 2012.

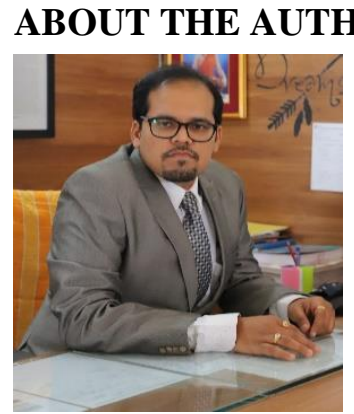

Prof. Sanjeevkumar Angadi has completed BE from Dayananda Sagar College of Engineering, Bengaluru and M. Tech from Gogte College of Engineering, Belagavi. Currently pursuing research from Visvesvaraya Technological University, Belagavi. He is working as Assistant Professor in Department of Computer Science and Engineering, MAEER's MIT College of Railway Engineering and Research, Barshi. His area of research is Computer Vision, Machine Learning. He has published more than 20 research papers in International and National Journals. $\mathrm{He}$ has attended National and International conferences. $\mathrm{He}$ has won best paper award at one of the International conference. He has one grant certificate of patent, Government of India. $\mathrm{He}$ has published Three Indian patents. $\mathrm{He}$ has one International grant certificate, Government of Australia $\mathrm{He}$ is life time membership of ISTE, IAENG, ISRD and Institute of Scholars. Received "Best Research Inspiration 2020" by IJMTST Research Excellence Awards, "Young Scientist Award" by International Scientist Awards on Engineering, Science and Medicine, 17th and 18th-Oct-2020, Kolkata, India, "Best Faculty Award" by CISR Foundation on 2nd International Academic and Research Excellence Awards 2020, "InSc Research Excellence Award 2020", "Best Teacher Award 2020" by High Range Book of World Records.

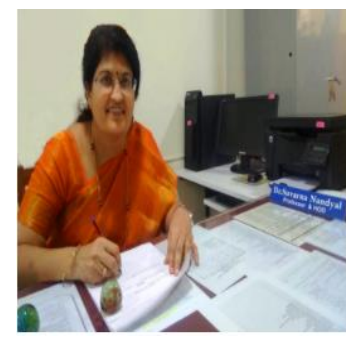

Dr. Suvarna Nandyal has completed BE and $\mathrm{M}$. Tech from Poojya Doddappa Appa College of Engineering Gulbarga and completed Ph.D. from JNT University, Hyderabad. She is working as Head of Department, Computer Science and Engineering. Her area of research is Digital Image Processing. She has published more than 80 research papers in International and National Journals. She has attended many national and international conferences. She has organized workshops on current topics like Digital Image Processing, Cloud computing and many more. She has presented a paper at International conference on Digital Image Processing at Singapore. She has one grant certificate of patent, Government of India. She has published five Indian patents. She has one International grant certificate, Government of Australia She is life time membership of ISTE, CSE, IEEE. 